\title{
Escuela de Gobierno basada en TIC: Determinante para la Accesibilidad e Integralidad del Empoderamiento Digital
}

Iris A. Jiménez-Pitre ${ }^{(1)}$, Raúl J. Martelo ${ }^{(2)}$ y José D.C. Jaimes ${ }^{(3)_{*}}$

(1) Universidad de la Guajira, Facultad de Ciencias Básicas y Aplicadas, Grupo de Investigación BIEMARC, Km 5 vía a Maicao, Guajira-Colombia.

(2) Univ. de Cartagena, Fac. de Ingeniería, Grupo de Investigación en Tecnologías de las Comunicaciones e Informática (GIMATICA), Avenida el Consulado, Calle 30 No. 48-152. Cartagena, Bolívar-Colombia.

(3) Universidad de Cartagena, Facultad de Enfermería, Grupo de Investigación en Medio Ambiente, Alimentos y Salud (MAAS), Cra. 50 \#24-120, Cartagena, Bolívar - Colombia, (e-mail:

jjaimesmor@yahoo.es)

*autor a quien debe ser dirigida la correspondencia

Recibido Oct. 6, 2016; Aceptado Dic. 6, 2016; Versión final Ene. 15, 2017, Publicado Oct. 2017

\section{Resumen}

En esta investigación se identifican las competencias tecnológicas de los funcionarios públicos colombianos del municipio de Riohacha a fin de detectar las brechas digitales que impiden el acercamiento de estos funcionarios a los ciudadanos. Con un enfoque metodológico cuali-cuantitativo, basado en un estudio de caso, se aplica un cuestionario a 35 representantes públicos del mencionado municipio. Los resultados cuantitativos se ubican en el nivel bajo, demostrando que casi nunca utilizan las potencialidades de las TIC para atender las necesidades de la población en términos de los servicios que ofrece el gobierno; mientras que, los cualitativos responden a características sociodemográficas de la muestra. En consecuencia, se propone una escuela de gobierno para la capacitación de dichos funcionarios en Tecnologías de Información y Comunicación con el fin de ofrecer mejor servicio a los ciudadanos.

Palabras clave: empoderamiento digital; accesibilidad digital; integración; escuela de gobierno; formación en TIC

\section{Government School based on ICT: A Key Strategy for Achieving Digital Empowerment, Accessibility, and Integrality}

\begin{abstract}
This research identifies the technological competencies of Colombian public officials in the municipality of Riohacha to detect the digital divide that prevents the approach of these officials to citizens. With a qualitative-quantitative methodological approach based on a study case, a questionnaire is applied to 35 representatives of the mentioned municipality. The quantitative results are located at the low level, showing that they almost never use the potential of ICT to meet the needs of the population in terms of the services offered by the government, while the qualitative data responds to the socio demographic characteristics of sampling. Consequently, a government school is proposed in this paper for the training these officials in Information and Communication Technologies, so they can offer better service to the citizens.
\end{abstract}

Keywords: digital empowerment; digital accessibility; integration; government school; ICT training 


\section{INTRODUCCIÓN}

A nivel mundial, las Tecnologías de la Información y la Comunicaciones (TIC) se han convertido en punta de lanza, tanto en la economía de regiones como en otras acciones humanas De Magdala y Pedruzzi (2013). Adicionalmente, las TIC representan un factor propulsor del desarrollo humano, debido a que han configurado un papel preponderante para el control de los gobiernos, mejora de prestaciones y apertura de espacios de participación, que podrían constituirse en herramientas útiles para contribuir a superar problemas existentes, en una sociedad democrática (Jiménez, 2014).

Corresponde a los líderes gubernamentales implementar mecanismos de gestión pública, para empoderar digitalmente a los ciudadanos en términos de accesibilidad e integración digital, con el fin de promover un gobierno eficaz y eficiente (Cano y Baena, 2015). Esto es posible al ofrecer servicios accesibles a la ciudadanía y permitir un mayor acceso a la información, a fin de transformar al gobierno en una institución competitiva y orientada al ciudadano (Piñero et al., 2007). Jornada (2001), afirma que los gobiernos a nivel mundial han variado respecto a iniciativas y acciones, al procurar siempre incorporar el uso intensivo de TIC en distintos ámbitos de los gobiernos para atender nuevas necesidades de democratización, rendición de cuentas y transparencia. La implementación de estos mecanismos digitales, planteado en Alcántara y Cendrós (2005), requiere que el gobierno tenga apertura a un modelo tecnológico de prestación de servicios en el que interactúen tanto niveles como las aristas del sistema de gobierno, lo que dinamiza la sociedad de la información a complejizar fenómenos como la globalización, virtualización, interactividad y construcción de nuevos espacios colectivos. Tal universalización de las relaciones sociales, de la información y del conocimiento en Castells (1997) se conoce como sociedad red.

Sobre la base de esta complejidad, resulta de especial relevancia el conocimiento y manejo de las TIC que puedan tener los líderes encargados de poner en marcha el modelo antes descrito. El desarrollo de competencias relacionadas con la accesibilidad e integración digital se convierten en dos de las principales dimensiones del empoderamiento digital que este líder debe dominar como parte de su formación integral, no sólo porque así lo exige la sociedad del conocimiento en la actualidad, sino porque también se verá incrementada la eficiencia de los procesos productivos, sus capacidades gerenciales y por ende la competitividad (Holmes, 2003).

Aún cuando el término empoderamiento data de los planteamientos emancipatorios de Freire (1966), quien no lo empleó para referirse al contexto digital sino que se centró en estudios de análisis de género, en la actualidad su significado se aborda desde distintos ángulos, coincidiendo en que es un intento de vencer estructuras opresoras individuales y sociales. Para efectos de esta investigación el empoderarse digitalmente implica poseer competencias digitales para vencer las brechas y desajustes sociales creados por la introducción de las TIC. Dichas competencias, se asumen como el conjunto de conocimientos, habilidades, actitudes, estrategias y sensibilización que se requieren cuando se utilizan las TIC y los medios digitales para realizar tareas, resolver problemas, comunicarse, gestionar información, colaborar, crear y compartir contenidos, construir conocimiento de manera efectiva, eficiente, adecuada, de manera crítica, creativa, autónoma, flexible, ética, reflexiva para el trabajo, el ocio, la participación, el aprendizaje, la socialización, el consumo y el empoderamiento (Ferrari, 2012).

A este complejo concepto, Serna (2016) agrega que las competencias digitales se conciben como una capacidad para la gestión del conocimiento tácito y explicito, empoderada por la utilización de las TIC y el uso estratégico de la información. Esta visión es reforzada por Cobo (2010) quien asegura que la e-competencia va más allá de la utilización de una TIC en particular, ya que también incluye conocimientos y actitudes orientadas al trabajo colaborativo, la innovación y el aprendizaje constante, así como la creación de nuevas ideas para enfrentar problemas desconocidos en diversos contextos. Por otro lado, según el Instituto de Tecnologías Educativas Departamento de Proyectos Europeos (2011) la competencia digital consiste en disponer de habilidades para buscar, obtener, procesar y comunicar información y para transformarla en conocimiento.

En lo que respecta al desarrollo efectivo de estas competencias, en el ámbito de las organizaciones públicas, Serna (2016) destaca que se requiere de transformaciones internas que permitan capacitar a sus funcionarios en el uso de las TIC para que contribuyan a la generación de un gobierno abierto como una nueva forma de participación y colaboración con la ciudadanía. En especial, a partir de las denominadas tecnologías sociales que favorecen la implicación de una ciudadanía activa. A tal efecto, Criado y Rojas (2013) se cuestionan si los servidores públicos están preparados para afrontar los nuevos retos que plantean las tecnologías y las nuevas formas de relación. En cuanto a la accesibilidad, es necesario que las tecnologías, los productos y los servicios TIC sean concebidos y diseñados de tal manera que permitan su utilización, interactuación y aportación por todas las personas que conforman la sociedad, sin distinguir sus capacidades o discapacidades. Desde el punto de vista del gobierno, tal accesibilidad se entiende como la garantía de contar con una red pública de banda ancha para el acceso a los servicios que ofrece el gobierno (Rodríguez y Silva, 2010). 
Ahora bien, considerar la accesibilidad desde la disponibilidad de una red banda ancha representa el elemento central de un nuevo sistema, caracterizado por complementariedades estructurales clave para el desarrollo económico y social. Esto se traduce en el eje de una dinámica que impacta al conjunto de la sociedad y a sectores productivos en un círculo virtuoso de desarrollo, basado en principios de eficiencia, innovación, colaboración e inclusión, propios de las redes (CEPAL, 2010). La misma se ha convertido, indiscutiblemente, en uno de las componentes estratégicos que soporta el crecimiento económico y social de los países. Esta red contribuye con la prestación de servicios electrónicos a la ciudadanía en materia de educación, salud y gestión gubernamental, lo cual permite optimizar esos servicios y superar barreras geográficas y financieras que restringen su cobertura a los segmentos pobres y marginados de la población (Jordán et al., 2010).

Estos segmentos de la población remiten obligatoriamente a puntualizar lo que se entiende por integración digital la cual, según Sánchez (2013), incluye el diseño de políticas que contribuyan a romper con aquellas asimetrías originadas por las brechas digitales e incluso que se puedan revertir a partir de la planificación e instrumentación de acciones estratégicas orientadas a colocar a los procesos informacionales de creación de conocimiento e innovación y de incorporación efectiva de las TIC al servicio de los más necesitados mediante procesos amplios de innovación, de participación y convivencia, y de creación de valor social para el desarrollo sustentable de la región y del país. Esto implica garantizar a los ciudadanos un gobierno electrónico que simplifique y profundice los requerimientos de relación y gestión con el propósito de lograr un país socio-productivo permeado por las TIC (Baena et al., 2014).

Dicha garantía demanda funcionarios públicos cuyas competencias tecnológicas, cognitivas y éticas, tal como las que proponen Esteve y Gisbert (2013), estén orientadas hacia: la búsqueda de nuevas metodologías, estrategias y técnicas para implementar políticas a través de las TIC; la planificación de la información; el manejo de plataformas multilenguaje; el uso de las redes sociales (blogs, twiter, skype, entre otros) para tener un mayor acercamiento con los ciudadanos, así como también resolver problemas legales y dilemas éticos mediante estas tecnologías (González y Sánchez, 2013). Es sobre todo un asunto de participación popular y requiere la toma en consideración de factores humanos como desencadenantes o no de los cambios indispensables para que el desarrollo tecnológico pueda ser aceptado, asimilado y adaptado por las mismas comunidades que se beneficiarán (Verdegem, 2011).

Dentro de estos factores humanos, Hill et al. (2008) sugieren que la edad, combinada con otros factores sociodemográficos como el estatus socioeconómico, seguirá siendo un factor clave que explicará significativamente la exclusión digital en el futuro, cuando las consecuencias de la misma sean más severas que ahora, ya que la tecnología afectará a cada aspecto cotidiano. En relación con la edad, Cresci et al., (2010) afirman que edades superiores usan menos Internet que los más jóvenes, siendo los hombres quienes usan significativamente más determinadas herramientas basadas en la web. Por otro lado, Peral et al (2013) en un estudio realizado sobre estas mismas variables sociodemográficas encontraron que a mayor formación académica y estrato social más alto, se reportaron mejores habilidades en el uso y manejo de las TIC. Estos autores concluyeron su investigación, demostrando que existen diferencias significativas en el empleo de algunas aplicaciones tecnológicas en función de las variables sociodemográficas sujetas a estudio.

En el caso de Colombia, a través del Plan Nacional Colombia Construye y Siembra Futuro (Colciencias, 2008), el gobierno reconoce a las TICs como herramientas que deben desarrollarse para la transferencia de información, lo cual significa su incorporación en las personas, en las organizaciones y en la sociedad en general. Es decir, más que un problema tecnológico de uso de nuevas tecnologías es un problema cognitivo. Este problema de conocimiento en el uso de las TIC también es destacado en las conclusiones presentadas por Montenegro y Niño (2001) como parte del Proyecto Andino de Competitividad. Entre las conclusiones que exponen estos autores acerca de la eficacia del gobierno en línea en Colombia, se encuentran la necesidad de hacer una mayor difusión de la Agenda de Conectividad, estimular la colaboración interinstitucional, mantener como punto de referencia el mundo y no sólo el ámbito local, a pesar de la tropicalización de tecnologías y procesos y hacer capacitación institucional.

Sobre la base de estas carencias cognitivas y de capacitación institucional, el objetivo de esta investigación fue proponer una escuela de gobierno para la formación en Tecnologías de Información y Comunicación (TIC), con el fin de ofrecer herramientas cognitivas necesarias para que funcionarios públicos colombianos puedan legislar sobre políticas públicas destinadas a la accesibilidad e integralidad del empoderamiento digital de los ciudadanos

\section{MATERIALES Y MÉTODOS}

Para el desarrollo de este trabajo se empleó un enfoque cuali-cuantitativo. El aspecto cualitativo se manifiesta a través de su diseño el cual se corresponde con un estudio de caso, definido por Neyman y 
Quaranta (2006) como aquel que recurre a diseños metodológicos que pueden combinar procedimientos cualitativos y cuantitativos y que tiene por objetivo el estudio de un sistema delimitado en tiempo y espacio de actores, relaciones e instituciones sociales. En este sentido, los datos se recogieron en el contexto geográfico del Departamento de La Guajira, tomando en consideración el número de actores o representantes gubernamentales vinculados con La Universidad de La Guajira.

Por otro lado, atendiendo al aspecto cuantitativo, en este estudio se utiliza una metodología de investigación descriptiva, no experimental, transeccional y de campo (Hernández et al., 2011). Dicha metodología se evidencia a través del conteo de los datos arrojados por la muestra para cuyo análisis se recurrió a la estadística descriptiva, mediante el uso de histogramas de frecuencias que ilustran el comportamiento de la media aritmética, como medida de tendencia central.

\section{Población y muestra}

Con base en el contexto geográfico descrito, la población la conforman 3 estratos, a saber 17 concejales de Riohacha, 9 diputados a la asamblea y 9 miembros del Consejo Superior Universitario (CSU), los cuales suman un total de 35 . En virtud de tratarse de un numero accesible o población finita, se decidió tomar a todos los sujetos como muestra, siguiendo así lo establecido por Castro (2003) que cuando la población es menor a 50, entonces esta población puede constituirse en muestra.

\section{Procedimiento para la recolección de información}

El procedimiento para la recolección de la información se dividió en dos fases. La primera se basó en la búsqueda y revisión de fuentes secundarias o documentales sobre los tópicos y temas relacionados con el empoderamiento y competencias digitales, así como también sobre propuestas vinculadas con la formación de personal en tecnologías de información y comunicación y la implementación en instituciones dedicadas a dicha formación. Para ello se tomaron, principalmente, los aportes hechos por Mejía y López (2016) acerca del Modelo de Calidad e-learning para Instituciones de Educación Superior en Colombia y los realizados por De Armas y De Armas (2011) sobre el Centro de Estudios de Administración Pública de la Universidad de La Habana (CEAP). La decisión de tomar a estos dos centros como modelos se debió a que el primero ofrece la metodología adecuada para la capacitación en TIC con las plataformas que cuenta el estado colombiano, y la segunda por la filosofía de gestión empleada en el ámbito de organizaciones públicas latinoamericanas.

La segunda fase, se destinó a determinar el nivel de conocimientos, habilidades y destrezas de los funcionarios públicos con respecto a la variable empoderamiento digital, para lo cual se diseñó un cuestionario contentivo de 20 preguntas. Dichas preguntas se confeccionaron a partir de la revisión teórica realizada sobre la variable en estudio la cual data de más de cuatro décadas, con los aportes de Freire (1966), hasta aquellos que definen el empoderamiento digital, incluyendo las dos dimensiones de accesibilidad e integración digital de Serna (2016), en este siglo. De estas 20 preguntas, 9 estuvieron relacionadas con la dimensión accesibilidad digital y 11 con integración digital. Previo a estas preguntas, se incluyeron los datos sociodemográficos de la muestra, referidos a edad, género, formación académica y estrato social. Este cuestionario fue sometido a la evaluación de expertos metodológicos y de contenido, arrojando un coeficiente de confiabilidad de 0,91. Dicho cuestionario se muestra en la Tabla 1.

Como puede observarse en la Tabla 1, la escala de frecuencias incluida en el Cuestionario contó con 5 opciones de respuestas con los siguientes valores: muy alta con escala de 5, alta con escala de 4, moderado con 3 , baja con 2 y muy baja con 1. Los intervalos utilizados para calcular la media de los resultados se presenta en la Tabla 2.

\section{Procedimiento para el análisis de los datos}

El análisis cualitativo de los datos reportados, en el primer apartado del cuestionario, requirió agrupar los cuestionarios atendiendo a las características sociodemográficas de la muestra. A tal efecto, se utilizó una matriz o tabla de registro que facilitó determinar las diferencias en cuanto al uso y manejo de las TIC, por edad, género, formación académica y estrato social. Para el análisis cuantitativo, se construyó la base de datos en una hoja de cálculo Excel 2013 para Windows en la cual se introdujeron las respuestas dadas por los funcionarios públicos seleccionados. Este procedimiento permitió calcular las frecuencias absolutas y relativas y los porcentajes correspondientes a las medias. Para ofrecer una mejor ilustración de los resultados arrojados por la muestra se recurrió al uso de histogramas de frecuencias. 
Tabla 1: Cuestionario para la recolección de datos

\begin{tabular}{|c|c|c|c|c|c|c|}
\hline $\begin{array}{l}\text { Datos } \\
\text { Personales }\end{array}$ & 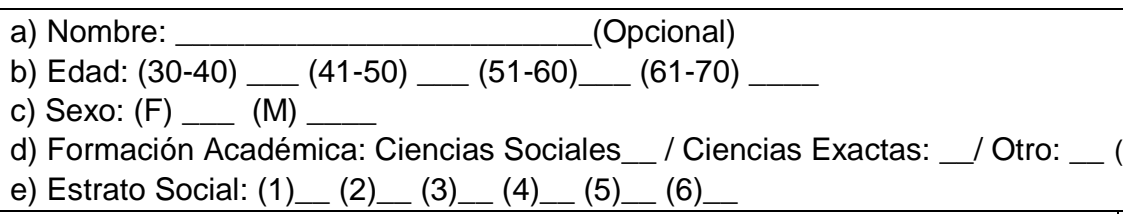 & $x p$ & que) & & & \\
\hline Dimensiones & Indicadores & 5 & 4 & 3 & 2 & 1 \\
\hline \multirow[t]{9}{*}{$\begin{array}{l}\text { Accesibilidad } \\
\text { digital }\end{array}$} & $\begin{array}{l}\text { La formación de los funcionarios públicos está basada en el uso de las TIC } \\
\text { para garantizar los servicios públicos. }\end{array}$ & & & & & \\
\hline & $\begin{array}{l}\text { Disponer de una red pública de banda ancha les permite a los ciudadanos } \\
\text { colombianos interactuar con los servicios que ofrece el Estado. }\end{array}$ & & & & & \\
\hline & $\begin{array}{l}\text { El uso de una red pública permite el desarrollo de las competencias } \\
\text { personales de los ciudadanos. }\end{array}$ & & & & & \\
\hline & $\begin{array}{l}\text { La formación de los funcionarios públicos a través de una red de banda ancha } \\
\text { impacta el crecimiento económico del país. }\end{array}$ & & & & & \\
\hline & $\begin{array}{l}\text { El disponer de una red pública de banda ancha genera procesos de } \\
\text { innovación. }\end{array}$ & & & & & \\
\hline & $\begin{array}{l}\text { La formación en gobierno electrónico eleva la calidad de los funcionarios en su } \\
\text { gestión. }\end{array}$ & & & & & \\
\hline & $\begin{array}{l}\text { Se desarrollan estrategias para la formación de funcionarios públicos que } \\
\text { garanticen la eficiencia y la eficacia en su gestión. }\end{array}$ & & & & & \\
\hline & $\begin{array}{l}\text { Colombia dispone de institutos de formación para el uso de las TIC dirigidos a } \\
\text { funcionarios públicos. }\end{array}$ & & & & & \\
\hline & Selecciona y discrimina la información en Internet. & & & & & \\
\hline \multirow[t]{11}{*}{$\begin{array}{l}\text { Integración } \\
\text { digital }\end{array}$} & $\begin{array}{l}\text { Busca nuevas metodologías, estrategias y técnicas para implementar políticas } \\
\text { públicas a través de las TIC. }\end{array}$ & & & & & \\
\hline & Identifica la comunicación sincrónica y la asincrónica. & & & & & \\
\hline & Planifica la información a través del manejo de las TIC. & & & & & \\
\hline & Proyecta videos para realizar presentaciones en su sitio de trabajo. & & & & & \\
\hline & Maneja diferentes plataformas para acercarse a los ciudadanos. & & & & & \\
\hline & Resuelve dilemas éticos con el uso de las TIC. & & & & & \\
\hline & Conoce y utiliza los aspectos legales para el uso de las TIC. & & & & & \\
\hline & Utiliza las redes sociales para comunicarse con los ciudadanos. & & & & & \\
\hline & Crea blogs para compartir información con los ciudadanos. & & & & & \\
\hline & Maneja el Skype como herramienta para comunicarse con la comunidad. & & & & & \\
\hline & Maneja el Twitter para conocer la opinión de los ciudadanos. & & & & & \\
\hline
\end{tabular}

Tabla 2: Intervalos para el cálculo de la media

\begin{tabular}{|c|l|l|l|}
\hline Valor & Intervalos & Alternativas & Categoría \\
\hline 5 & $4,21-5$ & Siempre & Muy alto \\
\hline 4 & $3,41-4,2$ & Casi siempre & Alto \\
\hline 3 & $2,61-3,4$ & Algunas veces & Moderado \\
\hline 2 & $1,81-2,6$ & Casi nunca & Bajo \\
\hline 1 & $1-1,8$ & Nunca & Muy bajo \\
\hline
\end{tabular}

\section{RESULTADOS Y ANÁLISIS}

Este apartado de presentación de los resultados con su respectivo análisis ha sido dividido en tres secciones, atendiendo al carácter cuali-cuantitativo de la investigación. En la primera, se reportan las características sociodemográficas de la muestra las cuales fueron extraídas de la primera parte del Cuestionario. Las secciones restantes están relacionadas con el análisis cuantitativo de las dos dimensiones del empoderamiento digital sometidas a evaluación, a saber, accesibilidad e integración digital.

\section{Características sociodemográficas de la muestra:}

Tal como se mencionó en los párrafos precedentes, la primera parte del Cuestionario se limitó a indagar información sobre los datos personales de la muestra, los cuales se convirtieron las características sociodemográficas que conforman este Estudio de Caso. La Tabla 3, reporta las características de los 35 funcionarios seleccionados para el desarrollo de esta investigación. 
Tabla 3: Características sociodemográficas de la muestra

\begin{tabular}{|c|c|c|c|c|c|c|c|}
\hline \multicolumn{2}{|r|}{ Edad } & \multicolumn{2}{|r|}{ Género } & \multicolumn{2}{|r|}{ Nivel Educativo } & \multicolumn{2}{|r|}{ Estrato social } \\
\hline No. & Rango & No. & Sexo & No. & Formación & No. & Nivel \\
\hline 10 & Entre 30 y 40 & 14 & Femenino & $20 \mid \begin{array}{ll}E \\
a \\
c \\
c\end{array}$ & $\begin{array}{l}\text { Egresados de Ciencias sociales: } \\
\text { abogados, contadores, administradores, } \\
\text { comunicadores sociales y trabajadores } \\
\text { sociales }\end{array}$ & 10 & 2 (bajo) \\
\hline 9 & Entre 41 y 50 & 21 & Masculino & 10 ir & $\begin{array}{l}\text { Egresados de Ciencias exactas: } \\
\text { ingenieros, médicos, }\end{array}$ & 15 & 3 (medio-bajo) \\
\hline 12 & $\begin{array}{l}\text { Entre } 51 \\
\quad \text { y } 60\end{array}$ & & & $5 E$ & Bachilleres académicos & 10 & 4 (medio) \\
\hline 4 & Entre 61 y 70 & & & & & & \\
\hline 35 & & 35 & & 35 & & 35 & \\
\hline
\end{tabular}

Como puede apreciarse en la Tabla 3, la mayoría (31) de los funcionarios públicos de la muestra cuentan con edades comprendidas entre los 30 a 60 años, prevaleciendo el género masculino con 21 representantes. De igual manera, 30 de estos funcionarios poseen estudios universitarios y 25 se ubican en un estrato social que va de medio-bajo a medio. Las habilidades y destrezas en cuanto al uso y manejo de las TIC se reportan en los párrafos siguientes.

\section{Accesibilidad digital}

Para proceder al análisis de esta dimensión, que incluyó nueve indicadores, a continuación se presentan la Tabla 4 y las Figuras 1 y 2 . Estas ilustraciones dan cuenta de los resultados de los 9 primeros indicadores dispuestos en el instrumento de recolección de datos.

Tabla 4: Resultados en frecuencias absolutas y relativas sobre la accesibilidad digital

\begin{tabular}{|c|c|c|c|c|c|c|c|c|c|c|c|c|c|c|c|c|c|c|}
\hline \multirow{3}{*}{$\begin{array}{l}\text { Dimensión } \\
\text { Indicadores }\end{array}$} & \multicolumn{18}{|c|}{ Accesibilidad digital } \\
\hline & \multicolumn{2}{|c|}{1} & \multicolumn{2}{|c|}{2} & \multicolumn{2}{|c|}{3} & \multicolumn{2}{|c|}{4} & \multicolumn{2}{|c|}{5} & \multicolumn{2}{|c|}{6} & \multicolumn{2}{|c|}{7} & \multicolumn{2}{|c|}{8} & \multicolumn{2}{|c|}{9} \\
\hline & $\mathrm{Fa}$ & $\mathrm{Fr}$ & $\mathrm{Fa}$ & $\mathrm{Fr}$ & $\mathrm{Fa}$ & $\mathrm{Fr}$ & $\mathrm{Fa}$ & $\mathrm{Fr}$ & $\mathrm{Fa}$ & $\mathrm{Fr}$ & $\mathrm{Fa}$ & $\mathrm{Fr}$ & $\mathrm{Fa}$ & $\mathrm{Fr}$ & $\mathrm{Fa}$ & $\mathrm{Fr}$ & $\mathrm{Fa}$ & $\mathrm{Fr}$ \\
\hline 1 & 3 & 8,6 & 11 & 31,4 & 9 & 25,7 & 5 & 14,3 & 8 & 22,9 & 9 & 25,7 & 3 & 8,6 & 14 & 40,0 & 12 & 34,3 \\
\hline 2 & 15 & 42,9 & 9 & 25,7 & 11 & 31,4 & 12 & 34,3 & 9 & 25,7 & 14 & 40,0 & 12 & 34,3 & 6 & 17,1 & 8 & 22,9 \\
\hline 3 & 10 & 28,6 & 7 & 20,0 & 6 & 17,1 & 9 & 25,7 & 10 & 28,6 & 7 & 20,0 & 9 & 25,7 & 8 & 22,9 & 6 & 17,1 \\
\hline 4 & 7 & 20,0 & 6 & 17,1 & 7 & 20,0 & 3 & 8,6 & 4 & 11,4 & 2 & 5,7 & 8 & 22,9 & 4 & 11,4 & 7 & 20,0 \\
\hline 5 & 0 & 0,0 & 2 & 5,7 & 2 & 5,7 & 6 & 17,1 & 4 & 11,4 & 3 & 8,6 & 3 & 8,6 & 3 & 8,6 & 2 & 5,7 \\
\hline Sumatoria & 35 & 100 & 35 & 100 & 35 & 100 & 35 & 100 & 35 & 100 & 35 & 100 & 35 & 100 & 35 & 100 & 35 & 100 \\
\hline Media por indicador & & 60 & & & 2, & 49 & 2,8 & & 2,6 & 63 & & 31 & $2, \varepsilon$ & 89 & & 31 & & 40 \\
\hline ledia de la dimensión & & & & & & & & & 2,5 & 54 & & & & & & & & \\
\hline
\end{tabular}

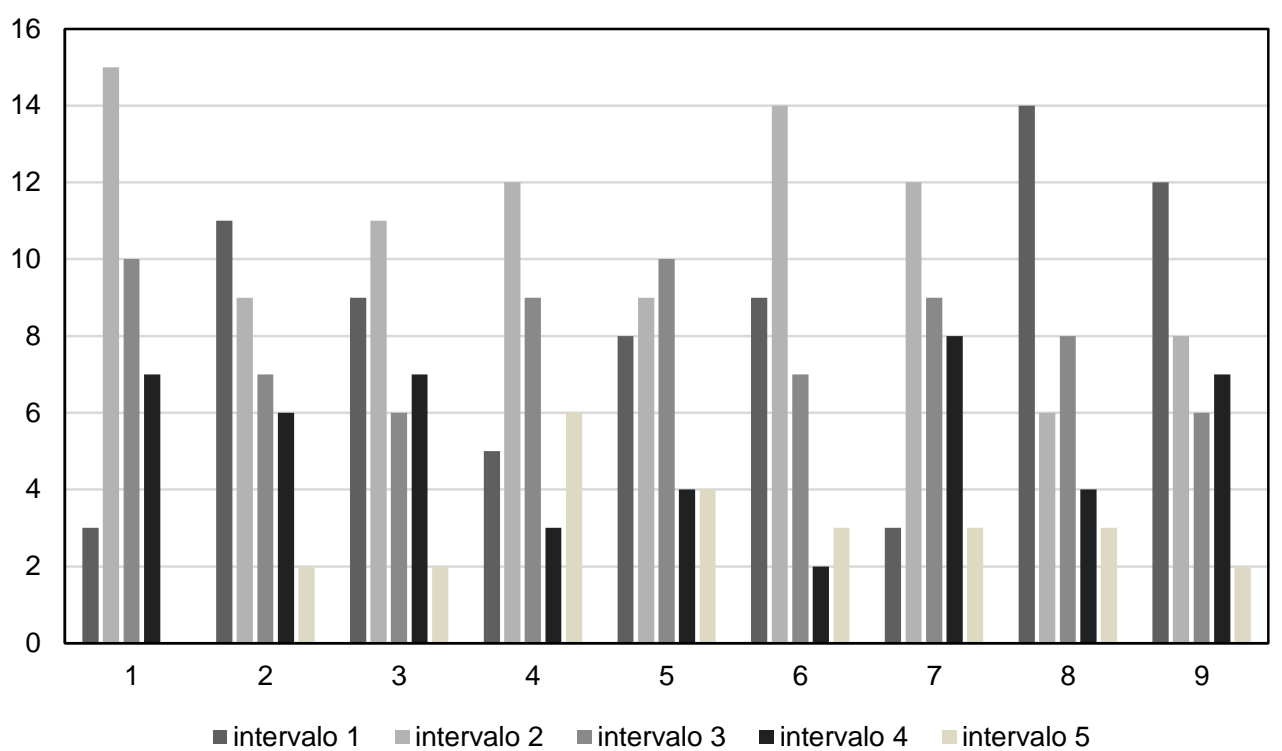

Fig. 1: Histograma de frecuencias absolutas sobre los nueve indicadores de la accesibilidad digital 


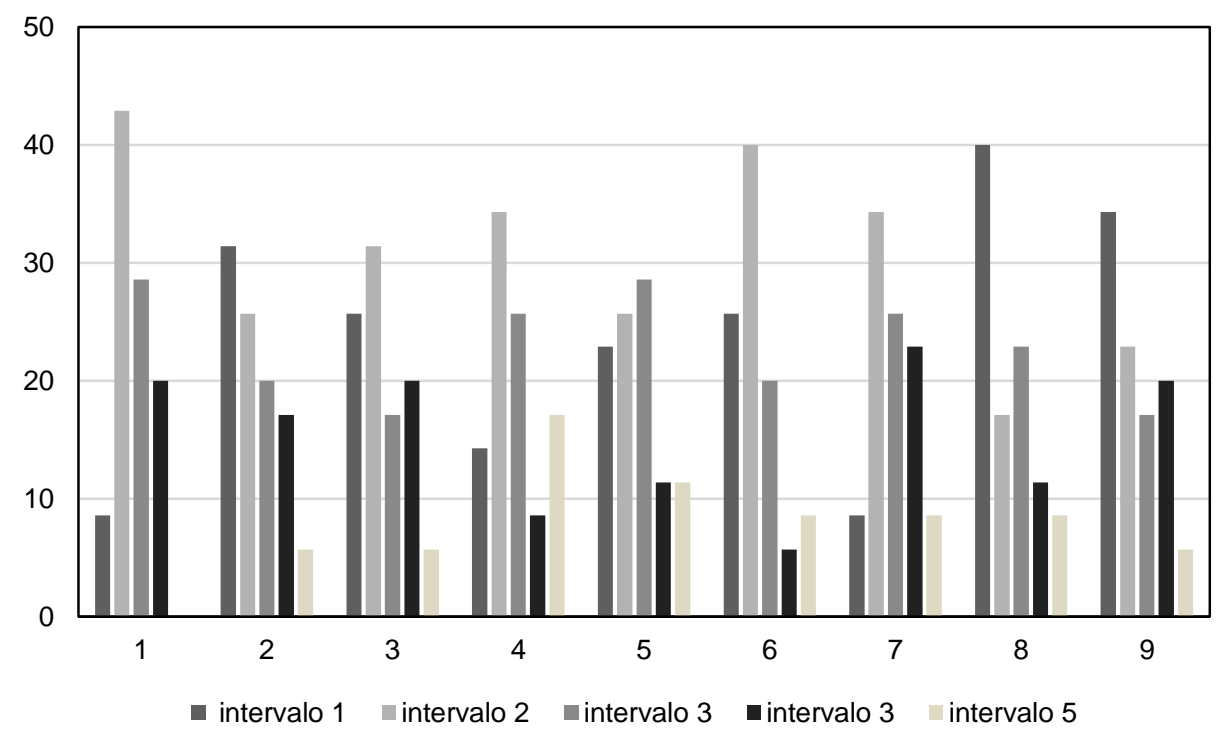

Fig. 2: Histograma de frecuencias relativas sobre los nueve indicadores de la accesibilidad digital

Al observar los resultados reportados por la muestra, se encontró que el promedio general de la media en esta dimensión de accesibilidad digital llegó a 2,54, ubicándose en la categoría bajo de acuerdo con el intervalo propuesto para tal fin. Así se ilustra en los histogramas de frecuencias (Figura 1 y Figura 2), cuyos máximos niveles no pudieron alcanzar la máxima categoría esperada, dada las acciones de reconocimiento de las TIC, plasmadas dentro del Plan Nacional Colombia Construye y Siembra Futuro (Colciencias, 2008). Esta ubicación conduce a reconsiderar los planteamientos hechos por Piñero et al., (2007) en cuanto a las responsabilidades de los líderes gubernamentales de implementar mecanismos para transformar las organizaciones públicas en instituciones más competitivas orientadas al ciudadano. Con esta ubicación, reportada en un nivel bajo, pareciera que los funcionarios públicos de la localidad aún se encuentran en el tránsito diferenciado puntualizado por Jornada (2001), en cuanto a la incorporación intensiva de las TIC en los distintos ámbitos de los gobiernos para atender las nuevas necesidades de democratización, rendición de cuentas y transparencia de las administraciones públicas.

Desde el punto de vista individual, al analizar las medias que condujeron a la ubicación general en la categoría bajo, se observó que los puntos más álgidos estuvieron constituidos por (1) la formación de los líderes en cuanto al gobierno electrónico (indicador 6) y (2) la disposición de institutos de formación en el país para el uso de las TIC (indicador 8); ambos se ubicaron en los niveles más bajos de la media, a saber, 2,31 . Con respecto al primer punto, los resultados demuestran la necesidad de enfatizar en la preparación de estos líderes en lo que concierne al uso estratégico e intensivo de las TIC, en particular de Internet, para ofrecer servicios públicos a los consumidores de una manera más adecuada, económica y conveniente, así como lo sugiere Holmes (2003), incluyendo la utilización de distintas herramientas, mecanismos y aplicaciones de las TIC por la administración pública como vía para la interconexión con otras organizaciones y personas, tal como lo proponen Criado et al., (2002). En relación con los resultados arrojados para el segundo punto, éstos reflejan la falta que hace en el país de una Escuela de Gobierno local en la cual se puedan adquirir las competencias digitales necesarias para que estos líderes públicos logren la universalización de las relaciones sociales, de la información y del conocimiento a los que hace alusión Castells (1997), para poderse insertar en lo que se denomina la sociedad red.

En atención a los indicadores encargados de medir la disposición de una red pública de banda ancha para interactuar con los servicios que ofrece el estado (indicador 2) y para el desarrollo de las competencias personales de los ciudadanos (indicador 3), lo cual a su vez le permite a los líderes seleccionar y discriminar la información en Internet (indicador 9), los porcentajes de la media se ubicaron también en un nivel bajo, reportando $2,40,2,49$ y 2,40, respectivamente. Vale la pena acotar que la carencia o el poco uso de esta red de banda ancha impide la dinámica establecida por la CEPAL (2010) que impacta al conjunto de la sociedad y a sectores productivos en un círculo virtuoso de desarrollo, basado en principios de eficiencia, innovación, colaboración e inclusión, propios de las redes.

El resto de los ítems incluidos en esta dimensión, destinados a indagar: la formación de los funcionarios públicos basado en las TIC (indicador 1), el desarrollo de estrategias que garanticen la eficiencia y la eficacia en su gestión (indicador 7), mediante una red de banda ancha para impactar el crecimiento del país (indicador 4) para así generar procesos de innovación (indicador 5), reportaron medias ubicadas en 2,60, 2,89, 2,80 y 2,63 , respectivamente. Al igual que para los indicadores anteriores, estos resultados entran en la categoría de 
bajo, permitiendo suponer dos tesis que pudieran estar influyendo en dichos resultados. La primera, relacionada con la poca apertura a un modelo tecnológico de prestación de servicios bastante complejo, en el que interactúan tanto los niveles como las aristas del sistema de gobierno, que plantean Alcántara y Cendrós (2005). La segunda, vinculada al desaprovechamiento por parte de estos funcionarios públicos de una red que contribuye con la prestación de servicios electrónicos a la ciudadanía en materia de educación, salud y gestión gubernamental, lo cual permite optimizar esos servicios y superar barreras geográficas y financieras que restringen su cobertura a los segmentos pobres y marginados de la población (Jordán et al., 2010).

En términos generales y de acuerdo con los resultados presentados, puede afirmarse que la concepción y diseño de las tecnologías, productos y servicios TIC dentro del contexto de investigación debe someterse a revisión ya que se está poniendo en riesgo la garantía de contar con una red pública de banda ancha para el acceso de la sociedad, sin distinguir sus capacidades o discapacidades, a los servicios que ofrece el gobierno (Rodríguez y Silva, 2010).

\section{Integración digital}

El análisis y discusión de los resultados sobre esta dimensión de integración digital se presentan en la Tabla 5 y se ilustran en las Figuras 3 y 4 de histogramas de frecuencias absolutas y relativas respectivamente.

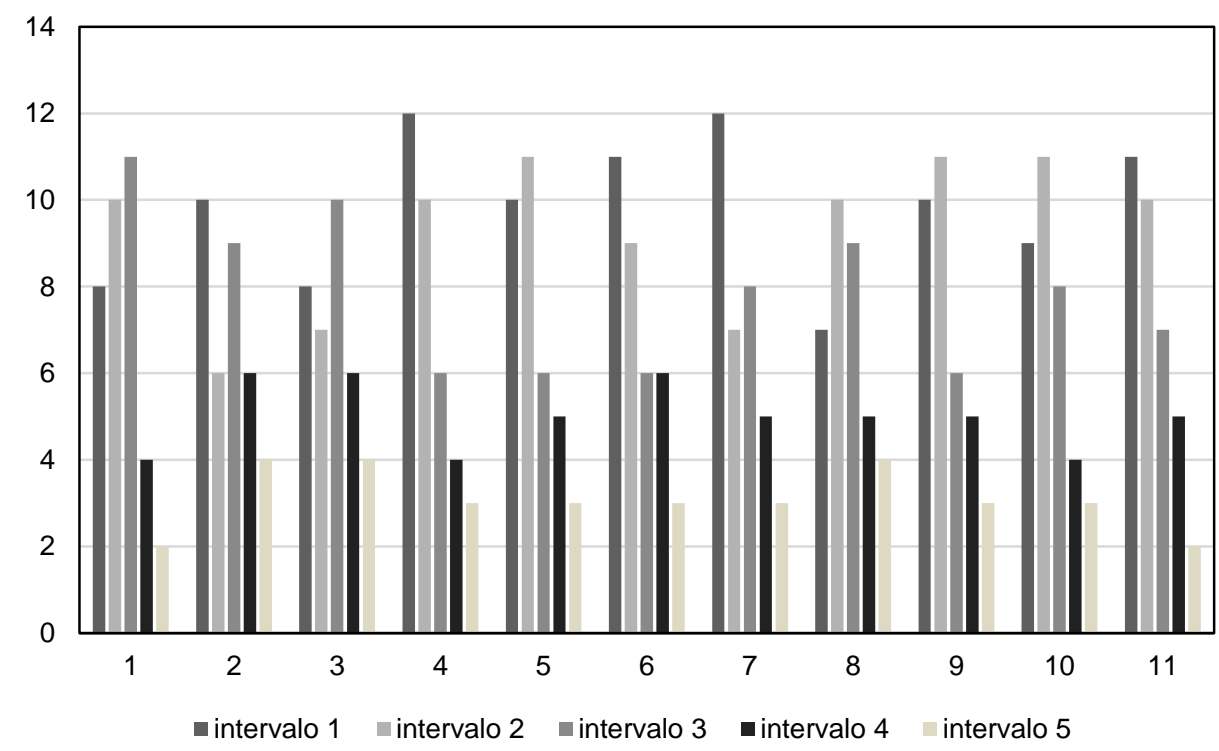

Fig. 3: Histograma de frecuencias absolutas sobre los once indicadores que midieron la integración digital

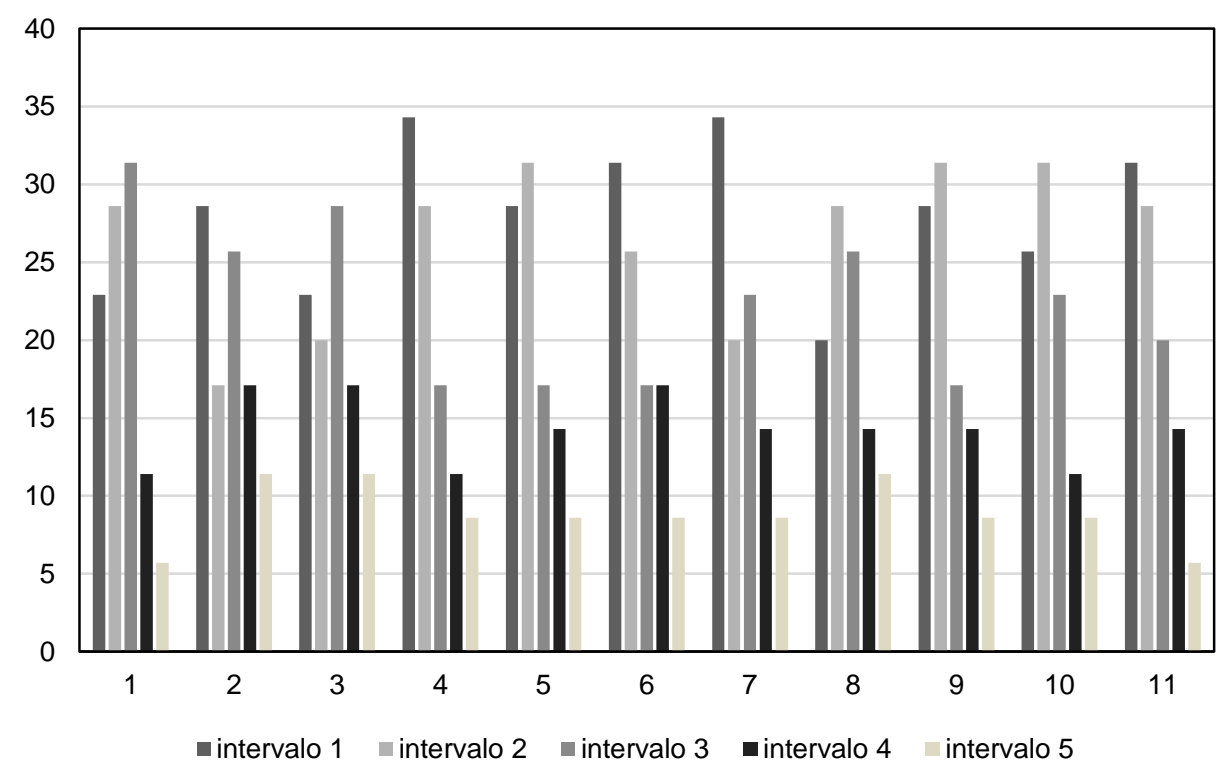

Fig. 4: Histograma de frecuencias relativas sobre los once indicadores que midieron la integración digital 
Tabla 5: Resultados en frecuencias absolutas y relativas sobre integración digital

\begin{tabular}{|c|c|c|c|c|c|c|c|c|c|c|c|c|c|c|c|c|c|c|c|c|c|}
\hline \multicolumn{22}{|c|}{ Integración digital } \\
\hline \multicolumn{2}{|c|}{10} & \multicolumn{2}{|c|}{11} & \multicolumn{2}{|c|}{12} & \multicolumn{2}{|c|}{13} & \multicolumn{2}{|c|}{14} & \multicolumn{2}{|c|}{15} & \multicolumn{2}{|c|}{16} & \multicolumn{2}{|c|}{17} & \multicolumn{2}{|c|}{18} & \multicolumn{2}{|c|}{19} & \multicolumn{2}{|c|}{20} \\
\hline $\mathrm{Fa}$ & $\mathrm{Fr}$ & $\mathrm{Fa}$ & $\mathrm{Fr}$ & $\mathrm{Fa}$ & $\mathrm{Fr}$ & $\mathrm{Fa}$ & $\mathrm{Fr}$ & $\mathrm{Fa}$ & $\mathrm{Fr}$ & $\mathrm{Fa}$ & $\mathrm{Fr}$ & $\mathrm{Fa}$ & $\mathrm{Fr}$ & $\mathrm{Fa}$ & $\mathrm{Fr}$ & $\mathrm{Fa}$ & $\mathrm{Fr}$ & $\mathrm{Fa}$ & $\mathrm{Fr}$ & $\mathrm{Fa}$ & $\mathrm{Fr}$ \\
\hline 8 & 22,9 & 10 & 28,6 & 8 & 22,9 & 12 & 34,3 & 10 & 28,6 & 11 & 31,4 & 12 & 34,3 & 7 & 20,0 & 10 & 28,6 & 9 & 25,7 & 11 & 31,4 \\
\hline 10 & 28,6 & 6 & 17,1 & 7 & 20,0 & 10 & 28,6 & 11 & 31,4 & 9 & 25,7 & 7 & 20,0 & 10 & 28,6 & 11 & 31,4 & 11 & 31,4 & 10 & 28,6 \\
\hline 11 & 31,4 & 9 & 25,7 & 10 & 28,6 & 6 & 17,1 & 6 & 17,1 & 6 & 17,1 & 8 & 22,9 & 9 & 25,7 & 6 & 17,1 & 8 & 22,9 & 7 & 20,0 \\
\hline 4 & 11,4 & 6 & 17,1 & 6 & 17,1 & 4 & 11,4 & 5 & 14,3 & 6 & 17,1 & 5 & 14,3 & 5 & 14,3 & 5 & 14,3 & 4 & 11,4 & 5 & 14,3 \\
\hline 2 & 5,7 & 4 & 11,4 & 4 & 11,4 & 3 & 8,6 & 3 & 8,6 & 3 & 8,6 & 3 & 8,6 & 4 & 11,4 & 3 & 8,6 & 3 & 8,6 & 2 & 5,7 \\
\hline 35 & 100 & 35 & 100 & 35 & 100 & 35 & 100 & 35 & 100 & 35 & 100 & 35 & 100 & 35 & 100 & 35 & 100 & 35 & 100 & 35 & 100 \\
\hline \multicolumn{2}{|c|}{2,49} & \multicolumn{2}{|c|}{2,66} & \multicolumn{2}{|c|}{2,74} & \multicolumn{2}{|c|}{2,31} & \multicolumn{2}{|c|}{2,43} & \multicolumn{2}{|c|}{2,46} & \multicolumn{2}{|c|}{2,43} & \multicolumn{2}{|c|}{2,69} & \multicolumn{2}{|c|}{2,43} & \multicolumn{2}{|c|}{2,46} & \multicolumn{2}{|c|}{2,34} \\
\hline \multicolumn{22}{|c|}{2,49} \\
\hline
\end{tabular}

Como puede observarse en ambas ilustraciones, los resultados de la dimensión integración digital, en términos del promedio general de la media, se ubicaron en 2,49 , situándose de acuerdo con el intervalo para su cálculo, en el nivel bajo. Al igual que en el caso anterior, resulta preocupante la valoración dada a esta dimensión por tratarse de la inclusión de políticas que contribuyen a romper las brechas digitales entre los ciudadanos, considerando aquellos menos aventajados para integrarlos digitalmente mediante procesos de participación, convivencia, y de creación de valor social para el desarrollo sustentable de la región y del país (Sánchez, 2013). Esto significa que queda en tela de juicio garantizar a los ciudadanos un gobierno electrónico que simplifique y profundice los requerimientos de relación y gestión con miras de lograr una región y, por ende, un país socio-productivo permeado por las TIC.

Tal como se ilustra en el histograma de frecuencias, es importante destacar que dentro de estos resultados se encontraron algunas medias en el nivel de moderado, específicamente, en aquellos casos relacionados con: la identificación de la comunicación sincrónica y asincrónica (indicador 11), la planificación de la información a través de las TIC (indicador 12) y el uso de las redes sociales para comunicarse con los ciudadanos (indicador 17) cuyos porcentajes fueron: 2,66; 2,74 y 2,69, respectivamente. Esta ubicación conlleva a inferir que la muestra posee conocimientos moderados acerca de lo que significa un gobierno interactivo, interjurisdiccional y totalmente conectado, así como lo define Dinsdale et al., (2002).

Desde el punto de vista cualitativo y con respecto a la caracterización sociodemográfica de esta muestra, ubicada en el nivel moderado como punto intermedio de la escala, se encontró que estos funcionarios pertenecen al género masculino, cuyas edades oscilan entre los 30 y 50 años, egresados de las Ciencias sociales y exactas, con estratos sociales que variaron de medio-bajo a medio. Estos resultados coinciden con los planteamientos hechos por Cresci et al., (2010) quienes afirman que en edades superiores, como por ejemplo el rango utilizado en esta investigación (de 51-60 y 61-70, usan menos Internet que los más jóvenes, siendo los hombres quienes usan significativamente más determinadas herramientas basadas en la web. De igual manera ocurrió con la formación académica y el estrato social, tal como lo aseveran Peral et al. (2013), a mayor formación académica y estrato social más alto, se desarrollan mejores habilidades en el uso y manejo de las TIC.

Sin embargo, esto se contradice con los resultados reportados para el resto de los indicadores $(10,13,14$, 15 y 16) incluidos en esta dimensión de integración digital cuyas medias se situaron en un nivel bajo, oscilando entre 2,31 y 2,46. Se evidencia, entonces, que los lideres públicos de la muestra casi nunca llevan a la práctica las competencias establecidas por González y Sánchez (2013) relacionadas con la búsqueda de nuevas metodologías, estrategias y técnicas para implementar políticas a través de las TIC; la planificación de la información; el manejo de plataformas multilenguaje; uso de las redes sociales (blogs, twiter, skype, entre otros) para tener un mayor acercamiento con los ciudadanos, así como también resolver problemas legales y dilemas éticos mediante estas tecnologías. El desarrollo de dichas competencias, debe formar parte del quehacer cotidiano del funcionario público, sobre el supuesto de que este líder cuenta con conocimientos adecuados para ponerlas en marcha.

Con relación a esta última aseveración y tomando en cuenta los resultados arrojados para ambas dimensiones del empoderamiento digital, ubicadas en un nivel bajo, pareciera que el problema deviene de lo reportado por Colciencias (2008) y Montenegro y Niño (2001) cuando reportan que no se trata de un problema de conocimientos tecnológicos de uso de nuevas tecnologías, sino más bien de un problema cognitivo. De allí que, la propuesta de una Escuela de Gobierno cuyo fin determinante consiste en ofrecer las herramientas cognitivas necesarias para que los funcionarios públicos colombianos puedan legislar, de manera adecuada, sobre políticas públicas destinadas a la accesibilidad e integralidad del empoderamiento digital de los ciudadanos, encuentre plena justificación. Dicha propuesta se presenta a continuación. 


\section{Propuesta de Escuela de Gobierno basada en TIC}

La propuesta que a continuación se presenta, tal como se mencionó en los párrafos precedentes se fundamenta en los aportes realizados por Mejía y López (2016) y De Armas y De Armas (2011). Según los primeros autores, en el contexto de Colombia la evaluación de calidad de un proyecto académico debe plantearse desde cinco dimensiones que encierran los dominios, tipos y entidades que expresa el modelo genérico de estándares, los cuales involucran: organización (misión y visión), procesos de enseñanza aprendizaje, desarrollo del e-learning, talento humano e Infraestructura. La explicación de estas dimensiones, aplicadas al contexto de Riohacha, se expone en esta propuesta. De los segundos autores, cuya experiencia con centros de formación para funcionarios públicos proviene de la Habana, Cuba, se toman como referentes para esta proposición la misión y visión, adaptadas al contexto de investigación.

\section{Lineamientos para la Escuela de Gobierno basada en TIC}

Misión: Formar y capacitar a funcionarios que ejercen cargos públicos de la administración pública central y de los gobiernos locales, principalmente del Municipio de Riohacha, en el uso y manejo de herramientas de tecnologías de información y comunicación para que puedan desarrollar competencias relacionadas con la accesibilidad e integración digital a objeto de incrementar la eficiencia de los procesos productivos, sus capacidades gerenciales y, por ende, la competitividad.

Visión: Contribuir con el proceso de formación continua de los funcionarios que ejercen cargos públicos de la administración pública central y de los gobiernos locales, principalmente del Municipio de Riohacha, ofreciéndoles las herramientas cognitivas necesarias para que estos funcionarios públicos colombianos puedan legislar, de manera adecuada, sobre políticas públicas destinadas a la accesibilidad e integralidad del empoderamiento digital de los ciudadanos. Ello con una visión perspectiva de los retos del desarrollo local, sobre la base de los principios modernos de la gestión pública que deben estar en correspondencia con las particularidades de la economía y sociedad colombianas y de las características del entorno internacional a que se enfrentan.

Procesos de enseñanza-aprendizaje: Dentro de estos procesos, resulta de especial relevancia incluir las competencias catalogadas por la muestra de investigación en un nivel bajo. De allí que, dentro de la estructura curricular del Programa a diseñar para esta Escuela merecen inclusión obligatoria las competencias relacionadas con la accesibilidad e integración digital, definidas y descritas en la Introducción de este trabajo de investigación. De igual forma, se considera aquellas dimensiones sugeridas por Esteve y Gisbert (2013) relacionadas con a) la dimensión tecnológica, centrada en la comprensión de situaciones comunes en las cuales las personas pueden encontrarse en la vida cotidiana o al trabajar con el computador, b) la dimensión cognitiva, en la que se abordan los aspectos relacionados con la búsqueda, el procesamiento y la gestión de la información, datos y fuentes de referencia, y c) la dimensión ética, referida a comportamientos más o menos adecuados en el uso de las tecnologías en general y de Internet en particular.

Desarrollo del e-learning: El desarrollo del e-learning debe ajustarse a los acelerados cambios tecnológicos por los que continuamente atraviesa la sociedad de la información y el conocimiento. Así mismo, tomar en cuenta los procesos de comunicación asincrónica y sincrónica entre el funcionario público con el resto de los sectores de la sociedad, los atributos del sitio web en lo que concierne a fuentes de información para acercarse a estos sectores y la constante evaluación de estas redes de comunicación.

Talento humano: En esta dimensión se propone diagnosticar el nivel de formación en TIC de los funcionarios así como también la continua capacitación del personal docente como apoyo tecnológico de los líderes en formación.

Infraestructura: Se propone disponer de una plataforma digital y una red de banda ancha a la que puedan tener acceso tanto los líderes en formación como la población. Ambos se constituyen en elementos clave del sistema caracterizado por complementariedades estructurales clave para el desarrollo económico y social.

\section{CONCLUSIONES}

Los resultados arrojados por la población, ubicados en el nivel bajo, demuestran serias carencias cognitivas de empoderamiento digital por parte de los funcionarios encuestados, evidenciando que casi nunca utilizan las ventajas y potencialidades de las TIC para atender las necesidades de los ciudadanos en términos de los productos y servicios que ofrece el gobierno. Así fue confirmado a través de las medias resultantes para las dimensiones de accesibilidad con un 2,54 y de integración digital con un 2,49. 
Para atenuar o resolver tales carencias, la propuesta de la Escuela de Gobierno basada en TIC esbozada en este estudio pretendió dotar a estos líderes públicos de las herramientas cognitivas necesarias para que desarrollen y coordinen acciones de carácter multi e interdisciplinarias en donde las TIC se presentan como soluciones del ecosistema digital. El trabajo y los desafíos que involucra el implementar propuestas como ésta son indudablemente enormes pero, una decidida voluntad política de ir adelante con nuevas estrategias, nuevas inversiones y nuevas capacidades en temas como la formación en tecnologías de información y comunicación, contribuirá con el desarrollo de las regiones, como Riohacha y por ende con todo el país. Esto requerirá de un estudio más profundo que permita interactuar con la muestra, a través de una investigación participativa, y así poder detectar de manera más específica las falencias en el uso de las TIC por parte de los funcionarios públicos. Dichos estudios a futuro pudieran diseñarse sobre la base de los resultados arrojados en esta primera aproximación al problema detectado, así como también con una mayor representación que diera más solidez a la creación de la Escuela de Gobierno.

\section{REFERENCIAS}

Alcántara, A. y J. Cendrós, Modelo de gobierno electrónico para la alcaldía rural del Municipio Autónomo Colón, Revista Electrónica de Estudios Telemáticos, 4(2), 75-93 (2005)

Baena, J. y otros tres autores, Uso de Tecnologías de Información y Comunicación para la negociación internacional ¿Ventaja para las empresas colombianas?, Revista Ciencias Estratégicas, 22(32), 1-29 (2014)

Cano, J.A. y J.J. Baena, Tendencias en el uso de las tecnologías de información y comunicación para la negociación internacional, Estudios Gerenciales, 31(136), 335-346 (2015)

Castells, M., La Era de la Información: economía, sociedad y cultura, $1^{\text {a }}$ Ed., 23-50, Alianza Editorial: Madrid, España (1997)

Castro, F., El proyecto de investigación su esquema de elaboración, 2ª Ed., 141-155. Editorial Uyapar: Caracas, Venezuela (2003)

CEPAL (Comisión Económica para América Latina y el Caribe), La hora de la igualdad: brechas por cerrar, caminos por abrir, Naciones Unidas, Santiago de Chile (2010)

Cobo, C., Cultura digital y nuevos perfiles profesionales: Desafíos regionales, en @TIC Revista d'Innovació Educativa, 5 (2), 1-7 (2010)

COLCIENCIAS, Colombia Construye y Siembra Futuro, Política Nacional de Fomento a la Investigación y la Innovación, (en la web: https://goo.gl/GFhLJL, acceso: 9 de septiembre 2016) (2016)

Cresci, M., H. Yarandi, y R. Morrell, The Digital Divide and Urban Older Adults, CIN: Computers, Informatics, Nursing, 28(2), 88-94 (2010)

Criado, G., M.C., Ramilo y M. Salvador, La necesidad de teoría (s) sobre gobierno electrónico, Una propuesta Integradora, XVI Concurso de Ensayos y Monografías del CLAD sobre Reforma del Estado y Modernización de la Administración Pública, "Gobierno Electrónico", 1-52, Caracas, Venezuela (2002)

Criado, J. y F. Rojas, Las redes sociales digitales en la gestión y las políticas públicas. Avances y desafíos para un gobierno abierto. Barcelona: Escola d'Administració Pública de Catalunya (2013)

De Magdala, M., y L. Pedruzzi, Hábitat Living Lab, red de innovación social y tecnológica, Revista Iberoamericana de Ciencia, Tecnología y Sociedad, 23(8), 135-135 (2013)

De Armas, R. y A. De Armas, Las TIC y el Desarrollo local: Papel en la formación de funcionarios públicos en la ciudad de La Habana, Revista Observatorio Iberoamericana del Desarrollo local y la Economía Social, 5(10), 1-16 (2011)

Dinsdale, G., S., Chhabra y J. Rath-Wilson, Guía práctica para el Gobierno Electrónico: cuestiones, impactos y percepciones: Para el Diálogo Regional de Política del Banco Interamericano de Desarrollo BID. Centro Canadiense de Gestión, (en la web: https://goo.gl/mo9f8e, acceso: 14 de agosto 2016) (2002)

Esteve, F. y M. Gisbert, Competencia digital en la educación superior: instrumentos de evaluación y nuevos entornos, Revista La Cuestión Universitaria, (7), 48-59 (2013)

Ferrari, A., Digital Competence in Practice: an analysis of Frameworks. Sevilla, JRC IPTS (2012) 
Freire, P. Pedagogía de la autonomía. Saberes necesarios y práctica educativa, Madrid: Siglo XXI (1966)

González, M. y J. Sánchez, Análisis de las estrategias del Gobierno colombiano para la inclusión de los ciudadanos en la Sociedad de la Información propuestas desde 2000 hasta 2011, Revista de Estudios Sociales, (4) 49, 133-146 (2013)

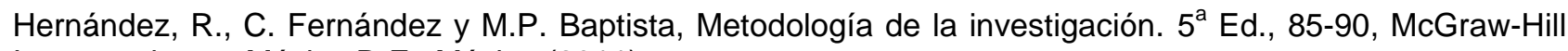
Interamericana, México D.F., México (2011)

Hill, R., Beynon-Davies, P. y Williams, M. Older people and internet engagement. Acknowledging social moderators of internet adoption, access and use, Information Technology \& People, 21 (3), 244-266 (2008)

Holmes, D., E-Gob. Estrategias para el uso eficiente del Internet en el gobierno, McGraw Hill, México D.F., México (2003)

Instituto de Tecnologías Educativas Departamento de Proyectos Europeos, Competencia digital, Ministerio de Educación, Gobierno de España, (en la web: https://goo.gl/xhTwMG, acceso: 9 de Diciembre 2016) (2011)

Jordán, V., W. Pérez y F. Rojas, Banda ancha: una urgencia para América Latina y el Caribe, Memorias del V Foro Ministerial Unión Europea - América Latina y el Caribe sobre la sociedad de la información, 21-37, Santiago de Chile (2010)

Jornada, J., Las administraciones públicas y la promoción de la sociedad de la información: opciones estratégicas y modalidades de intervención, Revista Gestión y Análisis de Políticas Públicas, CLAD, (16), 225-246 (2001)

Jiménez, G.A., Uso de las TICS en el aula de clases, escuelas del milenio como artefacto socio-técnico, estudio de caso de la unidad educativa del milenio "Bicentenario". Tesis de Maestría. Facultad Latinoamericana de Ciencias Sociales, Ecuador, Quito (2014)

Mejía, J. y D. López, Modelo de Calidad de E-learning para Instituciones de Educación Superior en Colombia, Formación Universitaria, 9(2), 59-72 (2016)

Montenegro, S. y L. Niño, Proyecto andino de Competitividad, La Tecnología de la Información y de las Comunicaciones en Colombia, Centro de Estudios de Desarrollo Económico Facultad de Economía Universidad de los Andes (2001)

Neiman, G. y G. Quaranta, "Los estudios de caso en la investigación sociológica". En: de Gialdino, Vasilachis (Comp.), 220. Estrategias de investigación cualitativa, Buenos Aires: Gedisa (2006)

Peral, B., J. Arenas y M. Ramón, El papel de las variables sociodemográficas en el uso de las aplicaciones basadas en Internet por los Mayores, Revista de Ciencias Administrativas y Sociales, 26 (62) 55-62 (2013)

Piñero, M. y otros tres autores, Premisas para una estrategia de gobierno electrónico en la gerencia de la virtualización universitaria, Revista Venezolana de Gerencia, 12(40), 572-598 (2007)

Rodríguez, J. y J. Silva, Incorporación de las tecnologías de la información y la comunicación en la formación inicial docente el caso chileno, Innovación Educativa, 6(32), 19-35 (2010)

Sánchez, S., La Integración Digital en el contexto de la Integración Regional: Consideraciones y propuestas. Sistema Económico Latinoamericano y del Caribe (SELA) (2013)

Serna, M. y O. Ramírez, Gobierno abierto y competencias digitales: Transformando la administración pública para afrontar los retos del nuevo paradigma, Cuadernos de Gobierno y Administración Pública, 3(1), 1-14 (2016)

Verdegem, P., Social media for digital and social inclusion: challenges for information society 2.0 research \& policies, Journal for a Global Sustainable Information Society, 9(1), 28-38 (2011) 